\title{
Emerging nanoparticulate drug delivery systems of metformin
}

\author{
Yao Chen ${ }^{1} \cdot$ Xinzhu Shan ${ }^{1} \cdot$ Cong Luo ${ }^{1} \cdot$ Zhonggui He $^{1}$
}

Received: 8 February 2020 / Accepted: 31 March 2020 / Published online: 6 April 2020

(c) The Author(s) 2020, corrected publication 2020

\begin{abstract}
Background Driving the conventional drug in new applications has emerged as a research hotspot for disease treatment. Metformin (MET) is conventionally used for the treatment of type II diabetes. It has also been found to be a versatile molecule with wide biological functions, such as losing weight. anti-aging and anticancer activity. Rational design of nanoparticulate drug delivery systems (nano-DDS) could significantly improve drug delivery efficiency. Recently, a wide range of nanoDDS has been developed to improve the delivery efficacy of MET or to perform as versatile nanoplatforms for efficient drug delivery.

Area covered In this review, we outline the emerging trends in advanced nano-DDS of MET, focusing on nano-DDS of MET for diabetes therapy, nano-DDS of MET for anticancer therapeutics, and nano-DDS of MET for other therapeutic aims. Expert opinion Despite the great progression in nano-DDS of MET, there's still a long way to truly put the conventional drug in new applications. Several important issues should be fully taken into consideration, such the manufacturing cost and economic burdens for patients, the biocompatibility and long-term toxicity of carrier materials, scale-up preparation difficulties, as well as the species gap between human beings and animal models.
\end{abstract}

Keywords MET $\cdot$ Nano-DDS $\cdot$ Diabetes $\cdot$ Anticancer $\cdot$ Versatile treatments

\section{Introduction}

Conventional drug in new applications has provided more versatile therapeutic options for clinical treatment of diseases (Baker et al. 2018). Among them, metformin (MET, dimethylbiguanide) has attracted considerable attention as a promising therapeutic agent for multiple diseases in recent years (Wang et al. 2017). MET is traditionally used for treatment of type II diabetes mellitus (Wang et al. 2017; Shurrab and Arafa 2020). MET, a cationic drug with a daily dose as high as several grams, which demonstrates well established efficacy and little toxicity (Wang et al. 2017; Shurrab and Arafa 2020). More interestingly, it has also been found to have multiple biological functions, such as losing weight, anti-aging and anticancer activity (Wang et al. 2017; Shurrab

Cong Luo

luocong@syphu.edu.cn

Zhonggui He

hezhonggui@vip.163.com

1 Department of Pharmaceutics, Wuya College of Innovation, Shenyang Pharmaceutical University, 103 Wenhua Road, Shenyang 110016, People's Republic of China and Arafa 2020). Particularly, patients with diabetes mellitus are at higher risk of cancer, MET has been found to demonstrate significant antitumor activity against a wide range of cancers (Samuel et al. 2019; Yu et al. 2019). Due to its cationic structure, the in vivo biomedical process of MET is significantly affected by organic cation transporters (OCTs) (Pakkir Maideen et al. 2017; Liang and Giacomini 2017). As a result, the expression heterogeneity of OCTs usually leads to great differences in oral bioavailability and therapeutic efficacy (Pakkir Maideen et al. 2017; Liang and Giacomini 2017). Therefore, it's necessary to develop suitable drug delivery systems (DDS) to achieve OCTs-independent delivery of MET.

Biomedical nanotechnology has made technological innovation in efficient delivery of drugs (Chen et al. 2018; van der Meel et al. 2019; Zhang et al. 2019; Shan et al. 2020). Formulating drugs into nanoparticulate drug delivery systems (nano-DDS) has demonstrated distinct advantages over traditional dosage forms (van der Meel et al. 2019). For oral drug delivery, encapsulation of drugs into nano-DDS could significantly improve dissolvability, stability and oral absorption efficiency (Luo et al. 2014a, b; Vllasaliu et al. 2018). Moreover, the oral absorption 
mechanisms of nano-DDS are quite different from free drugs in conventional formulations. Lymph absorption and endocytosis of nanoparticles (NPs) play more important roles in oral absorption of drugs into the blood (Luo et al. 2014a, b; Vllasaliu et al. 2018). For intravenous administration, nano-DDS could significantly change drug metabolic process in blood, due to the protection effect of nanocarriers and PEGylation modification (Chen et al. 2018; Luo et al. 2014a, b; van der Meel et al. 2019). Moreover, nano-DDS encapsulating anticancer drugs could significantly increase the drug accumulation in tumors owing to the well-known enhanced permeability and retention (EPR) effects or active targeting strategies (Luo et al. 2016a, b; Sun et al. 2019a, b). Additionally, subcutaneous administration of nano-DDS could achieve the aims of long acting and sustained release by controlling the drug release rate from nano-DDS (Luo et al. 2016a, b; Sun et al. 2018; Sun et al. 2019a, b). Therefore, nano-DDS has provided a versatile nanoplatform for efficient drug delivery.

In recent years, a wide range of nano-DDS was also developed for efficient delivery of MET. Rational design of nano-DDS could significantly improve the delivery efficiency of MET. Given great progress has been made in nano-DDS of MET, a wide range of nano-DDS has been developed to perform as versatile nanoplatforms for treatment of many diseases (Fig. 1). Therefore, it's timely to outline the recent progressions in emerging nano-DDS of MET. In this review, we summarized the emerging trends in advanced nano-DDS of MET, including nano-DDS of MET for diabetes therapy, nano-DDS of MET for anticancer therapeutics, and nano-DDS of MET for other therapeutic aims.

\section{Nano-DDS of MET for efficient diabetes treatment}

At present, diabetes is still one of the most serious chronic diseases threatening human health (Chaudhury et al. 2017; Chastain et al. 2019; Gao et al. 2019). MET, a biguanide therapy agent for diabetes, has been widely used for the treatment of type II diabetes due to its mild side effects (Wang et al. 2017; Shurrab and Arafa 2020). However, there's still some shortcomings of MET greatly limited its therapeutic efficiency, including insufficient intestinal absorption, high dose and frequent dosage administration requirement owing to its short half-life (Wang et al. 2017; Pakkir Maideen et al. 2017; Liang and Giacomini 2017). To respond to these disadvantages, several nano-DDS have been rationally designed and developed for efficient delivery of MET. Drug-encapsulated nano-DDS shows more properties of nanocarriers instead of the intrinsic properties of drugs (van der Meel et al. 2019). Therefore, nano-DDS could significantly change the way of drug delivery. A variety of MET-encapsulated nano-DDS have been contrasted by non-covalently loading MET into polymeric NPs, and various new polymers have been designed and synthesized for efficient delivery of MET. These MET-encapsulated nano-DDS could be approximately divided into three types: (i) MET-encapsulated nano-DDS for oral delivery; (ii) MET-conjugated nano-DDS for oral delivery; and (iii) MET-encapsulated nano-DDS for parenteral delivery.

\section{MET-encapsulated nano-DDS for oral delivery}

MET, a hydrophilic molecule with high solubility and poor absorption characteristics, belongs to Biopharmaceutics Classification System (BCS) class III drugs (Wang et al.

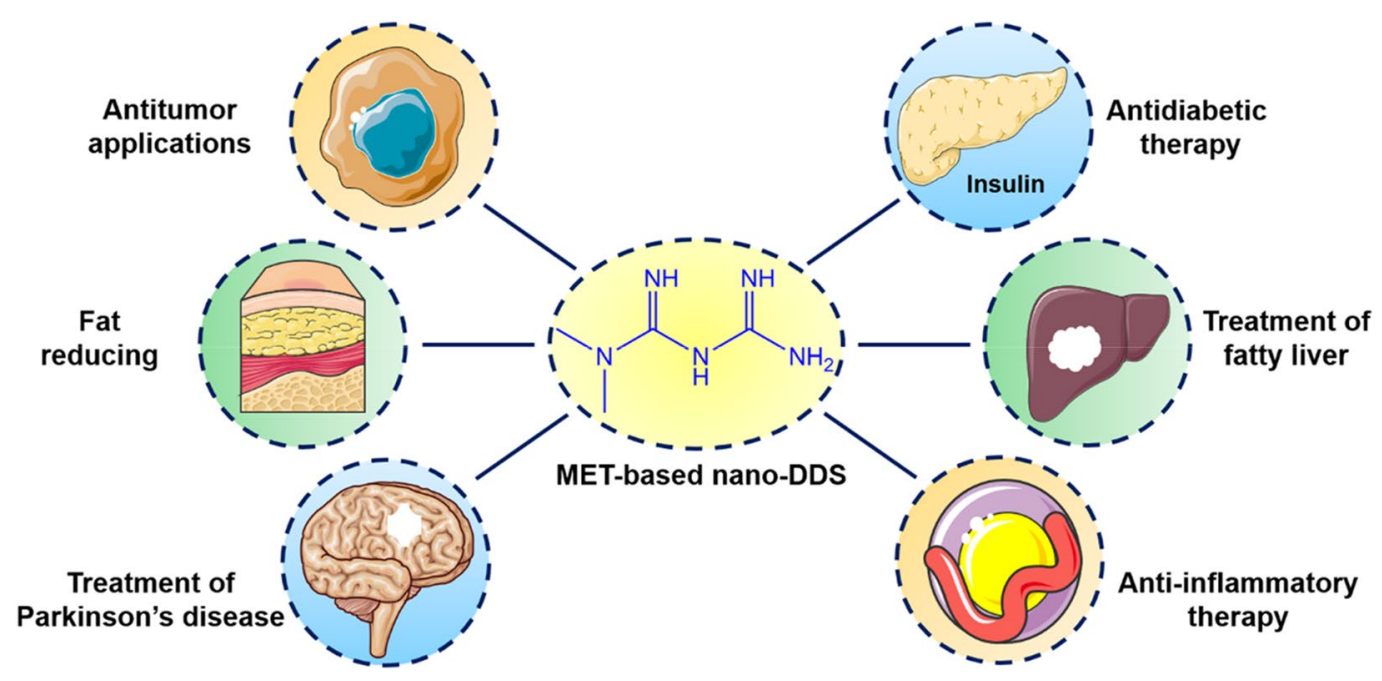

Fig. 1 Schematic representation of the versatile applications of nano-DDS of MET 
2017; Shurrab and Arafa 2020). The unsatisfactory oral absorption efficiency and short half-life of MET lead to frequent dosage administration with high dose, even gram levels one day (Wang et al. 2017; Shurrab and Arafa 2020). Therefore, great efforts have been made to improve this situation, and several nano-DDS loaded with MET were rationally designed to obtain controlled drug release and improved oral absorption, including alginate NPs (Kumar et al. 2017), alginatechitosan NPs (Mokhtare et al. 2017), hyaluronic acid NPs (Bhujbal and Dash 2018) and chitosan/ eudragit nanocomplex (Sahu and Verma 2016). For instance, MET-loaded alginate NPs were developed by an emulsion cross-linking technology for diabetes treatment (Kumar et al. 2017). The gastrointestinal absorption and intestinal permeability of MET were significantly improved when compared with free drug (Kumar et al. 2017). As a result, low dose of MET-loaded alginate NPs $(46.8 \mathrm{mg} / \mathrm{kg})$ produced comparable hypoglycaemic effects with high dose of free MET $(150 \mathrm{mg} / \mathrm{kg}$ ) (Kumar et al. 2017). The advantages of METloaded nano-DDS for oral drug delivery should be attributed to the sustained drug release and enhanced gastrointestinal permeability, resulting in significant decrease dosage of MET (Cui et al. 2018; Patiño-Herrera et al. 2019).

\section{MET-conjugated nano-DDS for oral delivery}

Non-covalent encapsulation of drugs into nano-DDS has some disadvantages, including low drug loading efficacy, poor stability and premature drug leakage from the nanocarriers (Luo et al. 2014a, b). Therefore, development of new-type nano-DDS based on conjugation chemistry has become a research hotspot, including MET-conjugated nanoDDS for oral delivery (Mirazi et al. 2015). For instance, Mirazi et al. developed MET-conjugated nanotubes for the control of blood glucose level in a streptozotocin-induced diabetic rat model (Mirazi et al. 2015). Carbon nanotubes have been found to possess the advantage to pass through the plasma membrane with negligible damage to the cells, resulting in improved absorption efficacy and reduced side effects (Mirazi et al. 2015). In this study, the authors compared the blood glucose homeostasis of free MET and MET-conjugated carbon nanotubes in diabetic rats induced by intraperitoneal administration of streptozotocin $(60 \mathrm{mg} /$ $\mathrm{kg}$ ) (Mirazi et al. 2015). The rats were orally administrated with free MET or MET-conjugated nanotubes $(150 \mathrm{mg} / \mathrm{kg})$ daily and every $48 \mathrm{~h}$ for 1 week (Mirazi et al. 2015). When compared with free MET, MET-conjugated nanotubes demonstrated much better therapeutic efficacy by reducing the blood glucose levels in diabetic rats (Mirazi et al. 2015). The good therapeutic efficacy should be attributed to the good stability and high oral absorption of MET-conjugated nanotubes in gastrointestinal tract (Mirazi et al. 2015).
MET-encapsulated nano-DDS for parenteral delivery

Moreover, MET-loaded nanofilms and microneedles were also widely investigated for parenteral delivery in the treatment of diabetes (Sarwar et al. 2020; Shariatinia and Zahraee 2017; Yu et al. 2017). For instance, Sarwar et al. designed a novel smart nanofilm by mixing chitosan with polyethylene glycol methyl ether (PEGME) for controlled drug release of MET (Sarwar et al. 2020). Similarly, Shariatinia and Zahraee developed a biocompatible nanofilm using chitosan and poly(ethylene glycol)-block-poly(propylene glycol)-blockpoly(ethylene glycol) (BP) polymers for controlled release of MET (Shariatinia and Zahraee 2017) (Fig. 2). MET showed a rapid release characteristic within $24 \mathrm{~h}$ and a sustained release behavior for 15 days (Shariatinia and Zahraee 2017). Such a unique drug release property could be utilized for sustained drug delivery of MET in parenteral administration (Shariatinia and Zahraee 2017). In addition to nanofilms, Yu et al. designed near-infrared light triggered microneedles for transdermal delivery of MET in treatment of diabetes (Yu et al. 2017). Photothermal conversion Prussian blue NPs and MET were embedded in the microneedles, and the arrowheads of microneedles were capped on polyvinyl alcohol/ polyvinyl pyrrolidone (PVA/PVP) as solid supporting substrate (Yu et al. 2017). Under near-infrared light (NIR) irradiation, the arrowheads melted, facilitating rapid release of encapsulated MET (Yu et al. 2017). Therefore, such a lighttriggered release characteristic of MET could be utilized to achieve precisely topical therapy of diabetes (Yu et al. 2017).

Despite the great progression of nano-DDS of MET for diabetes treatment, formulating MET into nanocarriers will inevitably increase manufacturing complexity and costs, which could pose an obstacle to potential clinical translation. Comparatively speaking, MET-microneedles represent the most promising perspective formulations for topical administration of MET, owing to the long acting and sustained releasing characteristics of microneedles. There's no doubt that application of nanotechnology in MET-based diabetes treatment could significantly improve the therapeutic efficacy. However, the potential clinical application of METbased diabetes treatment should fully balance advantages and drawbacks of nanotechnology-driven diabetes therapy in the future.

\section{Nano-DDS of MET for cancer treatment}

Compared with diabetes therapy, nano-DDS have been more commonly developed for anticancer therapy, and several nano-formulations have been utilized in clinical cancer treatment (Luo et al. 2012). Biomedical nanotechnology could significantly facilitate the anticancer efficacy of therapeutic agents by improving their physicochemical properties, extending their circulation time in blood and accelerating 
Fig. 2 Mesoporous MCM-41 NPs-containing chitosan-based nanocomposite films loading permission, from (Shariatinia and Zahraee 2017). Copyright (2017), Elsevier Ltd with MET. Reproduced, with
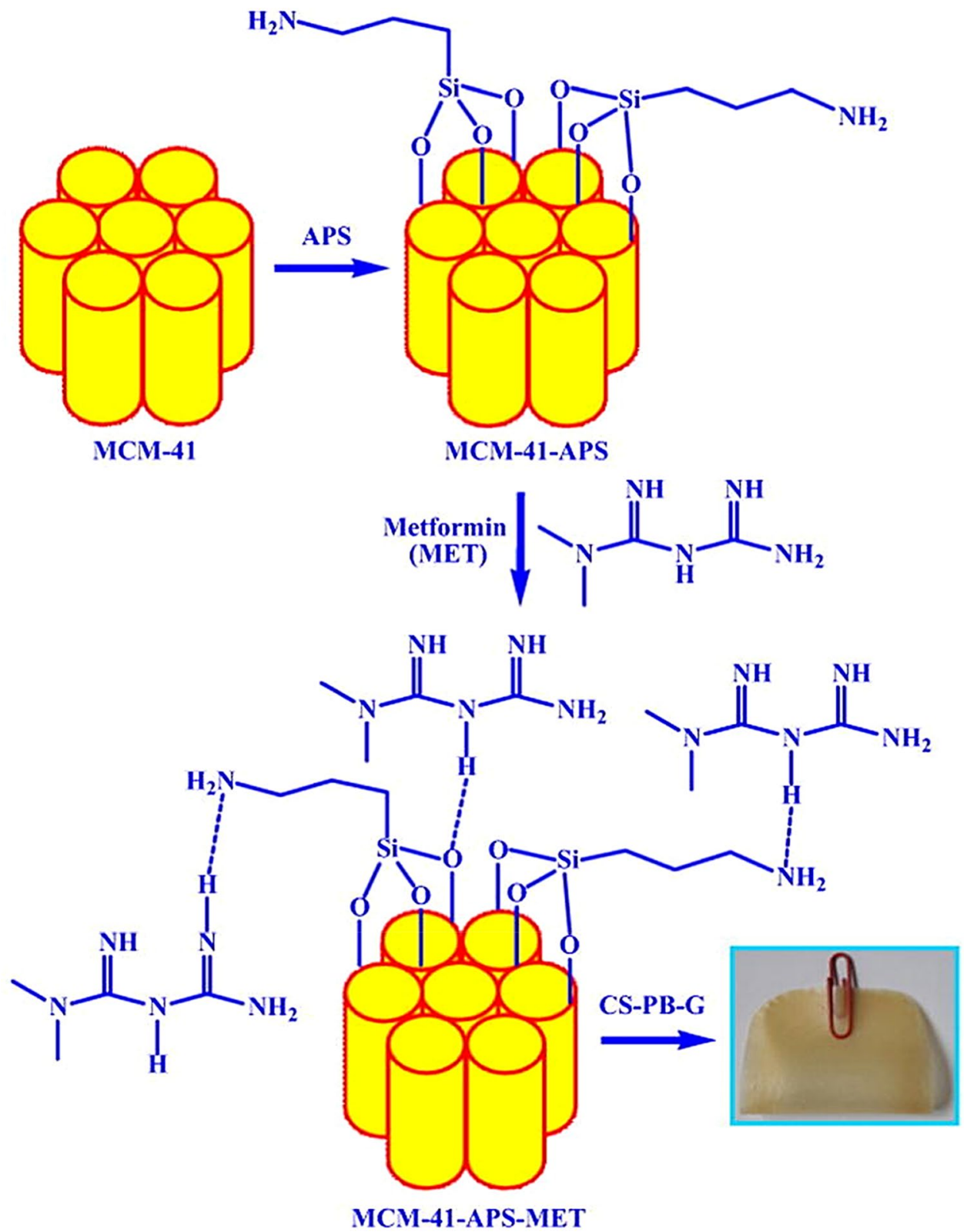

their tumor accumulation (Chen et al. 2018; van der Meel et al. 2019; Zhang et al. 2019). The recent discovery of MET with anticancer activity has made it a hot drug for cancer treatment (Samuel et al. 2019; Yu et al. 2019). As a promising anticancer agent, a wide range of preclinical and clinical studies have been carried out on MET (Samuel et al. 2019; Yu et al. 2019). Inhibition of mitochondrial complex I and activation of AMPK are the main anticancer mechanisms of MET (Samuel et al. 2019; Yu et al. 2019; Morales and Morris 2015). Moreover, targeting epigenetic regulation and acting on the cancer stem cells (CSCs) were also found to be involved in the anticancer activity of MET, which are closely associated with the drug resistance, tumor metastasis and recurrence (Samuel et al. 2019; Yu et al. 2019; Morales and
Morris 2015). Additionally, MET was also found to have the capacity of radiosensitization in anticancer treatment (Samuel et al. 2019; Yu et al. 2019; Morales and Morris 2015). In this section, we will discuss the emerging nano-DDS of MET for cancer treatment, including: (i) MET-encapsulated nano-DDS for cancer therapy; (ii) MET-derivative-based nano-DDS for cancer therapy; and (iii) nano-DDS of MET for combination cancer therapy.

\section{MET-encapsulated nano-DDS for cancer therapy}

In recent years, a variety of MET-encapsulated nanocarriers have been developed for anticancer therapy, including liposomes, nanomicelles, polymeric NPs and inorganic 
NPs. Among them, MET-encapsulated liposomal vesicles were widely investigated (Lee et al. 2020; Magdy et al. 2017; Osama et al. 2019; Shukla et al. 2019). For instance, Shukla et al. prepared MET-encapsulated liposomes with enhanced therapeutic efficacy against breast cancer (Shukla et al. 2019). Thin-film hydration method was utilized to prepare the liposomes (Shukla et al. 2019). Three drug loading methods were used to encapsulate MET, including passive loading, active loading, and drug-loaded lipid film (Shukla et al. 2019). The drug-loaded film method showed distinct advantages over the other two loading methods in terms of entrapment efficiency (Shukla et al. 2019). The MET-encapsulated liposomes demonstrated significantly improved cellular uptake, cytotoxicity and antimetastatic activity against breast cancer (Shukla et al. 2019). In addition, another METencapsulated liposomal vesicle, sterosomes, was constructed for the treatment of lung cancer (Osama et al. 2019). METloaded sterosomes demonstrated dose and time-dependent inhibitory effect against A549 cells (Osama et al. 2019). Compared with MET solution, MET-loaded sterosomes significantly extended the circulation time of MET in the blood (Osama et al. 2019). Therefore, MET-encapsulated liposomal vesicles have showed great potential in clinical treatment of cancers.

In addition to liposomal vesicles, MET-encapsulated nanomicelles and NPs were also widely investigated for cancer therapy (Arafa et al. 2018; Javidfar et al. 2018; Jose et al. 2015). For instance, Arafa et al. designed a MET-encapsulated hybrid nanocarrier for adjunct treatment of colon cancer, in which the nanocarrier was fabricated by incorporating chitosan-based semi-interpenetrating network into Pluronic nanomicelles (Arafa et al. 2018). The MET-encapsulated hybrid nanocarrier showed a sustained drug release characteristic within $48 \mathrm{~h}$ and proved sensitization of colon cancer treatment (Arafa et al. 2018). Moreover, MET-attached nanosystems were also utilized for anti-metastatic therapy (Nurani et al. 2017). For instance, Nurani et al. designed MET-modified cellulose nanofibers by attaching MET on the surface of cellulose nanofibers via electrostatic interaction (Nurani et al. 2017). The FTIR spectroscopy and zeta potential changes of nanofibers confirmed the success attachment of MET (Nurani et al. 2017). The prepared MET-attached nanofibers significantly prevented the invasion and migration of melanoma cells, demonstrating promising application in the inhibition of melanoma metastasis (Nurani et al. 2017).

In addition to the above organic NPs, polymers-modified gold NPs were also utilized for efficient delivery of MET in cancer therapy, including chitosan (Aldea et al. 2018), hyaluronic acid (Kumar et al. 2015) and dextran (Qian et al. 2017). For instance, Qian et al. developed sugar-coated core-shell nanobullet with gold core and dextran shell, and MET was interspersed in the dextran shell (Qian et al.
2017). The dextran shell significantly promoted the cellular uptake of gold NPs, with the subsequent release of MET (Qian et al. 2017). Compared with free MET or bare gold NPs, the sugar-coated core-shell nanobullet showed much more potent cytotoxicity against MCF-7 breast cancer cells (Qian et al. 2017). And MET-induced antitumor mechanisms were found to be related to the inactivation of mitochondria and activation of p53 protein (Qian et al. 2017). Similarly, Kumar et al. also developed a hyaluronic acid-functionalized gold nanocomplex for targeting delivery of MET in liver cancer therapy (Kumar et al. 2015). Green synthesis method was utilized to fabricate the gold NPs, in which the extract of Solanum melongena $L$ was used as reducing agent (Kumar et al. 2015). Then, the gold NPs was capped with hyaluronic acid for targeting drug delivery, and MET was loaded in the hyaluronic acid-functionalized gold nanocomplex (Kumar et al. 2015). Such a hybrid nano-formulation showed targeted delivery of MET in HepG2 cells with significantly increased regression activity when compared with free MET (Kumar et al. 2015).

Therefore, various MET-encapsulated nano-DDS have been developed for anticancer therapy, including organic and inorganic nanocarriers. Formulating MET into suitable nanocarriers could significantly improve the drug delivery efficiency of MET, including prolonging circulation time in blood, facilitating tumor accumulation, increasing cellular uptake, and improving cytotoxicity against different kinds of tumor cells. As a result, MET-encapsulated nano-DDS showed distinct advantages over free MET in efficient cancer therapy. Additionally, MET-encapsulated nano-DDS was also widely utilized for combination cancer therapy, which will be discussed in the section of "Nano-DDS of MET for combination cancer therapy".

\section{MET derivative-based nano-DDS for cancer therapy}

As previously discussed, MET has a very simple chemical structure with the main structure of biguanide (Wang et al. 2017; Shurrab and Arafa 2020). More particularly, the biguanide group of MET is the key moiety for its pharmacological activities (Wang et al. 2017; Shurrab and Arafa 2020). Therefore, the derivatives of MET could retain anticancer activity as potent as free MET, which makes MET a more significant attraction to be utilized in anticancer treatment. In recent years, several MET derivatives were rationally designed for the construction of a wide range of multifunctional nanocarriers in cancer therapy, including simple amphipathic MET derivative (Jiang et al. 2019), polymeric derivatives of MET (Ramasamy et al. 2019; Xiong et al. 2016; Zhao et al. 2016) and MET-based cationic lipids (Luo et al. 2016a, b; Shi et al. 2017).

All these MET derivative-based nano-DDS not only retained antitumor activity, but also participated in the 
construction of nanostructures for multifunctional applications. For instance, Jiang et al. synthesized an oleate carbon chain derivative of MET by simply conjugating dicyandiamide with oleyl amine (Jiang et al. 2019). As shown in Fig. 3, they prepared a hybrid nanomicelles assembled from the oleate carbon chain derivative of MET and docosahexaenoic acid, and the nanomicelles were further modified with fucoidan for targeting delivery (Jiang et al. 2019). The prepared nanosystem showed capacity in prolonging blood circulation and remodeling the inflammation microenvironment in premetastatic site, which resulted in potent inhibition of the adhesion of circulating tumor cells to the activated endothelial cells (Jiang et al. 2019). When combined with chemotherapy, the nanosystem inhibited both primary tumor progression and metastasis formation (Jiang et al. 2019).
Moreover, polymeric derivatives of MET were also rationally designed for efficient drug delivery in cancer treatment (Ramasamy et al. 2019; Xiong et al. 2016; Zhao et al. 2016). For instance, Leaf Huang's group designed a polymeric construction of MET (PolyMet) by conjugation of polyethylenimine (PEI) with dicyandiamide for efficient siRNA delivery (Zhao et al. 2016). The delocalized cationic charges in the biguanide groups of PolyMet significantly improved the safety of PEI, and PolyMet could be utilized to formulate siRNA into lipid-polycation-hyaluronic acid (LPH) NPs for systemic gene delivery (Zhao et al. 2016). More importantly, even without loading siRNA, the blank LPH-PolyMet NPs showed similar anticancer activity with MET by activating the AMPK and inhibiting the mTOR pathways (Zhao et al. 2016). Moreover, PolyMet were also utilized as both anticancer agent and carrier material for

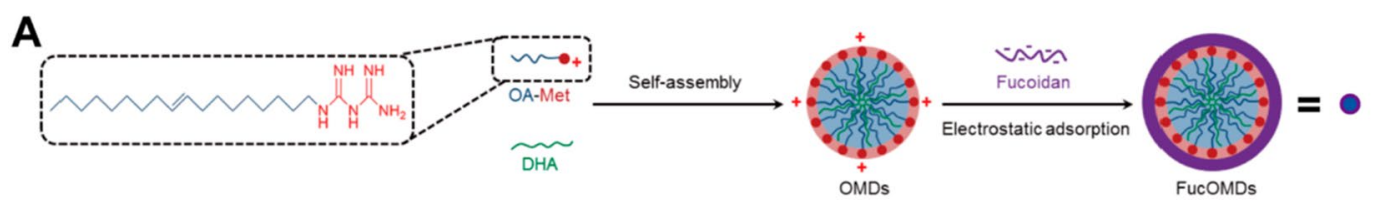

B

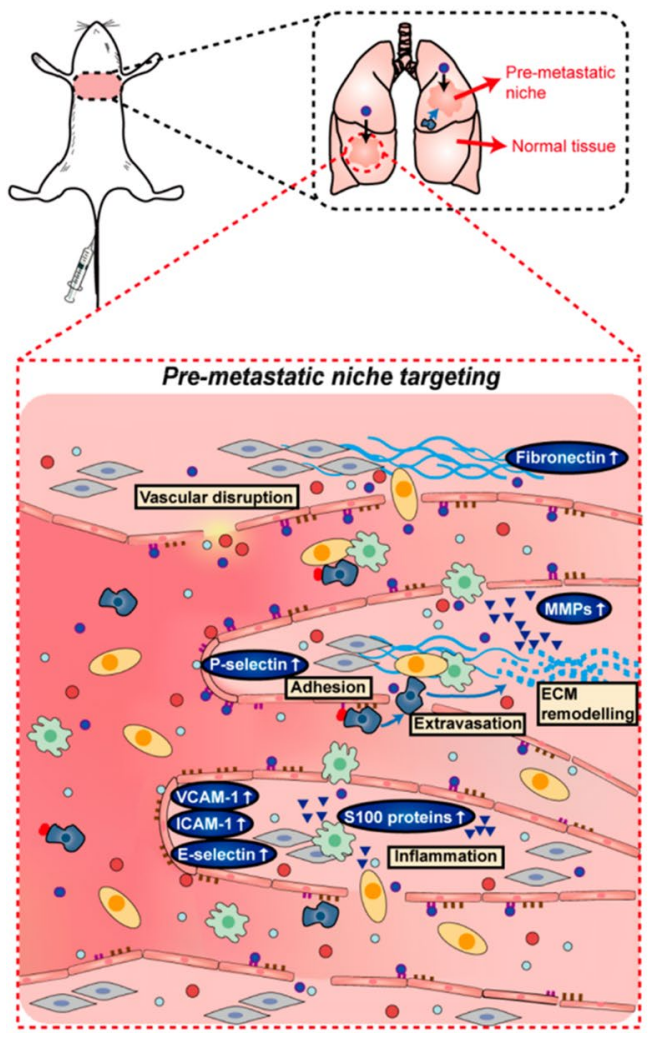

Fig. 3 Fucoidan-decorated self-assembly micelles delivering metformin and DHA (FucOMDs) for premetastatic niche-targeting and modification. a FucOMDs preparation. b Process of FucOMDs targeting P-selectin in the premetastatic microenvironment and the

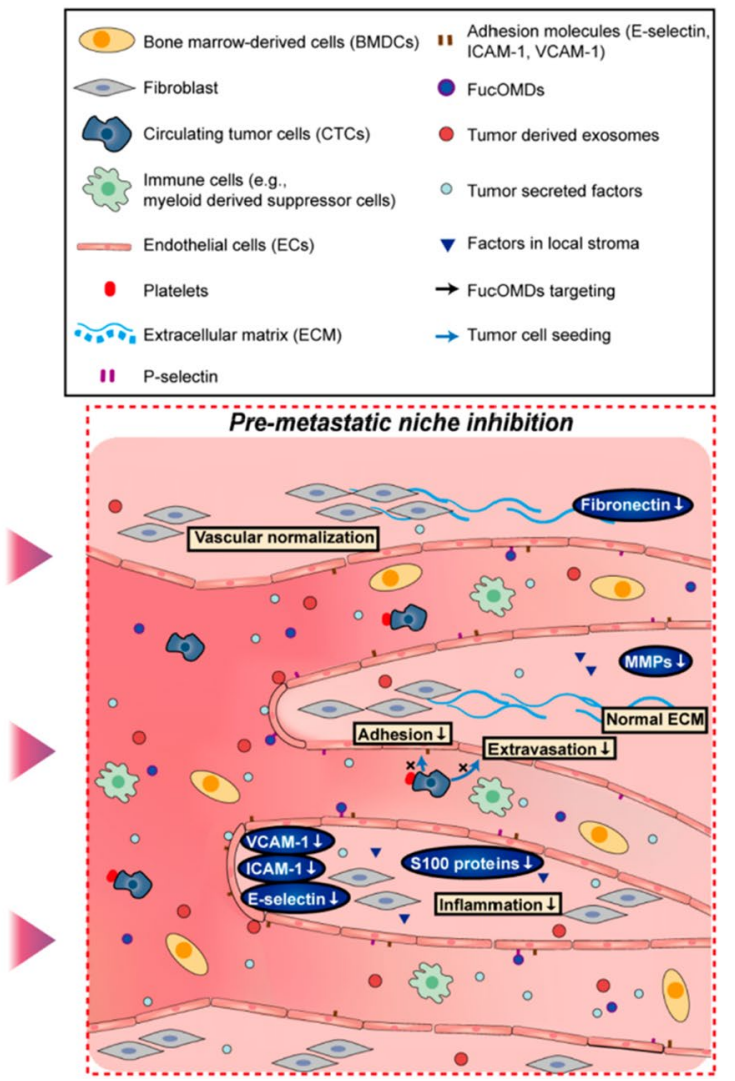

inhibitory effect against typical aberrant alterations in the microenvironment by FucOMDs. Reproduced, with permission, from (Jiang et al. 2019). Copyright (2019), American Chemical Society 
combination cancer therapy with cisplatin (Xiong et al. 2016). Additionally, a polypeptide (poly-l-lysine [PLL]) derivative of MET (LysMET) with anticancer activity was synthesized and utilized as a gene carrier for efficient cancer therapy (Ramasamy et al. 2019).

In addition to polymeric derivatives of MET, METbased cationic lipids have also been rationally designed for multifunctional applications (Luo et al. 2016a, b; Shi et al. 2017). As shown in Fig. 4, a new cationic lipid (1,2-di-(9Zoctadecenoyl)-3-biguanide-propane (DOBP)) was synthesized using biguanide as the cationic head group (Luo et al. 2016a, b). The liposomes of DOBP showed much stronger anticancer activity when compared with a very similar cationic lipid (DOTAP), and the antitumor mechanism of DOBP-liposomes was very similar to MET (Luo et al. 2016a, b). More importantly, DOBP could help formulating TNF-related apoptosis-inducing ligand (TRAIL) plasmids into Lipid-Protamine-DNA (LPD) NPs for systemic gene delivery, and DOBP-LPD-TRAIL NPs showed synergetic anticancer activity by virtue of the intrinsic antitumor activity of DOBP and the therapeutic effect of TRAIL (Luo et al. 2016a, b). Similarly, another multifunctional MET-based cationic lipid was designed by conjugating MET with cholesterol (Shi et al. 2017). The cholesterol derivative of MET with intrinsic antitumor activity could also been utilized in constructing liposomal nanocarriers for combination of gene silencing and tumor growth inhibition (Shi et al. 2017).

\section{Nano-DDS of MET for combination cancer therapy}

The therapeutic efficacy of single drug is far from satisfactory due to the limited antitumor effect of one drug, drug resistance and tumor heterogeneity (Luo et al. 2019). Particularly, the anticancer activity of MET depends on regulation of the cellular metabolic pathways (Wang et al. 2017; Shurrab and Arafa 2020). As a result, the antitumor activity of MET is not as strong as most conventional chemotherapeutic agents. But MET has a satisfactory safety,
Fig. 4 a Chemical structures of DOTAP, metformin and DOBP. b Schematic illustration of LPDTRAIL NPs. Reproduced, with permission, from (Luo et al. 2016a, b). Copyright (2016), Elsevier Ltd

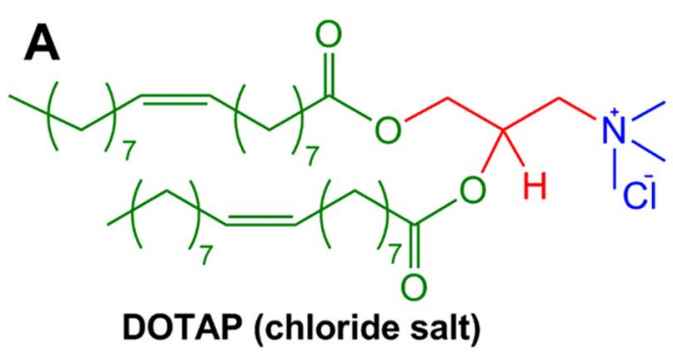<smiles>CN(C)C(=N)NC(=N)N</smiles>

Metformin hydrochloride

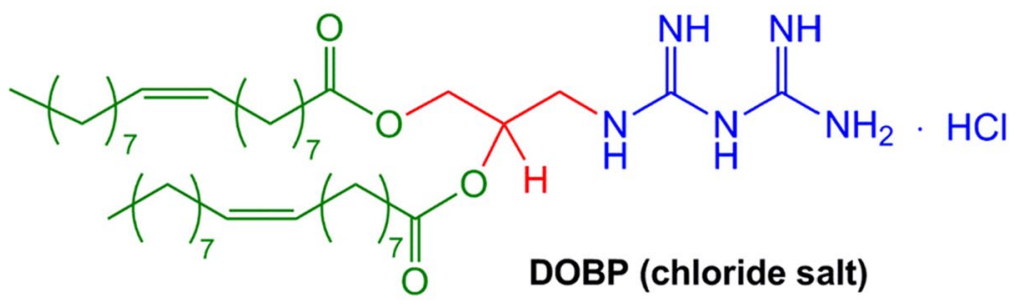

B

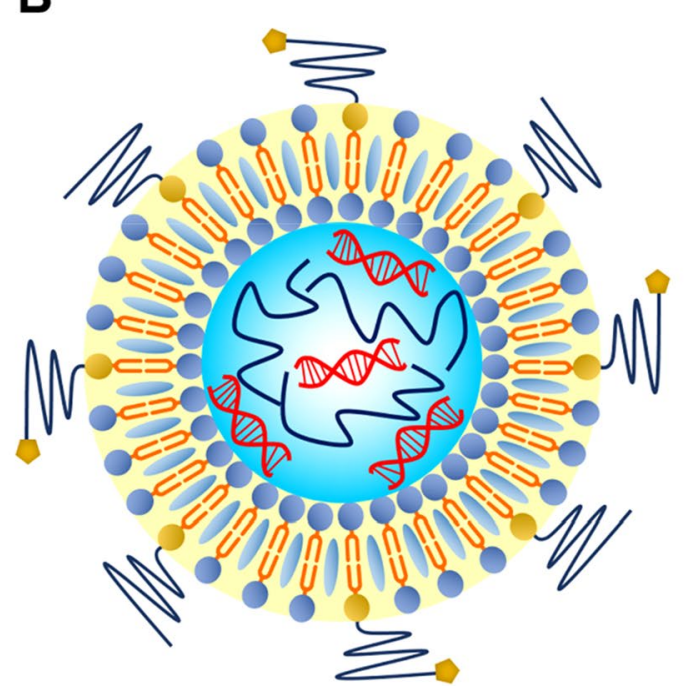

DSPE-PEG

DSPE-PEG-AA

DOBP (DOTAP)

Cholesterol

TRAIL DNA

Protamine 
combination cancer treatment using MET and other therapeutic agents could not only achieve synergistic therapeutic effect, but also guarantee the controllable side effects. Therefore, MET-based combination cancer therapy has been widely investigated in recent years, especially along with the rapid development of nano-DDS. These combos mainly included combination of MET with chemotherapeutic drugs, gene drugs and photosensitizers.

Chemotherapy represents the most used non-surgical therapeutic regimen in clinical cancer treatment (Siegel et al. 2020). A wide range of studies have been carried out to combine MET with clinical commonly used chemotherapeutic drugs to improve their therapeutic efficacy and attenuate their toxicity (Peng et al. 2017). Peng et al. summarized a diversity of molecular mechanisms of MET involved in the combination therapy with different chemotherapeutic drugs, mainly including AMPK/mTOR signaling pathway activation, suppression of HIF-1, p-gp and MRP1 protein expression, regulation of inflammatory signaling pathway, inhibition of oxidative stress, and induction of apoptotic mitochondria and nucleus (Peng et al. 2017). Several chemotherapeutic drugs have been utilized to combine with MET, including cisplatin, curcumin (Farajzadeh et al. 2018), doxorubicin (Li et al. 2019; Shafiei-Irannejad et al. 2018), paclitaxel (Xiao et al. 2018), irinotecan (Taghizadehghalehjoughi et al. 2018) and topotecan (Banala et al. 2018). And various nanocarriers have been designed for co-delivery of MET and chemotherapeutic drugs, such as liposomes, nanomicelles and polymeric NPs. These nanocarriers could not only realized highly efficient co-delivery of two drugs, but also could achieve synergistic treatment via regulation of precisely drug release and modulation of tumor microenvironments.

In addition to combining with chemotherapeutic drugs, a wide range of cationic derivatives of MET with intrinsic antitumor activity were designed to efficiently deliver gene drugs (DNA and RNA) for combination cancer therapy (Ramasamy et al. 2019; Xiong et al. 2016; Zhao et al. 2016; Luo et al. 2016a, b; Shi et al. 2017). As previously discussed, these cationic derivatives or lipid derivatives of MET not only retained the antitumor activity of MET, but also the biguanide structure of MET endow them with the capacity for efficient delivery of gene drugs (Ramasamy et al. 2019; Xiong et al. 2016; Zhao et al. 2016; Luo et al. 2016a, b; Shi et al. 2017). The polymeric derivatives of MET (PolyMet and LysMET) could form stable nanocomplexes with RNAs due to the interactions of positive/negative changes, and the nanocomplexes could be further formulated into liposomal NPs for efficient delivery of gene drugs (Ramasamy et al. 2019; Xiong et al. 2016; Zhao et al. 2016). Similarly, lipid derivatives of MET (e.g. DOBP) could assemble into liposomes, which could be utilized to formulating DNA plasmid for efficient drug delivery (Luo et al. 2016a, b; Shi et al. 2017). All these cationic MET derivatives-based
nano-DDS could achieve potent synergistic cancer treatment by virtue of their intrinsic antitumor activity.

Moreover, although photodynamic therapy (PDT) has been widely investigated as a non-invasive therapeutic approach of cancers, the therapeutic effect of PDT is greatly impeded by tumor hypoxia (Sun et al. 2020). MET has been found to improve tumor oxygenation, and cope with the hypoxic tumor environment (Song et al. 2017). Therefore, combination of MET with photosensitizer could achieve synergistic therapeutic effect. For instance, Song et al. developed a liposomal vehicle for efficient co-delivery of MET and chlorin e6 (Ce6, a commonly-used photosensitizer) (Song et al. 2017). MET and Ce6 were co-loaded into the inner hydrophilic cavity and outer hydrophobic bilayer of membrane of the liposomes, respectively (Song et al. 2017). The liposomes showed high cellular uptake and sustained release of MET, which could significantly improve tumor oxygenation (Song et al. 2017). As a result, combination of MET and Ce6 using liposomes distinctly improved the antitumor efficiency when compared with Ce6 alone (Song et al. 2017). Therefore, co-encapsulation of MET and photosensitizer into nanocarriers represents an efficient strategy to facilitate PDT efficiency by remodeling tumor oxygenation.

\section{Nano-DDS of MET for other therapeutic aims}

As previously discussed, MET is a unique therapeutic agent with versatile clinical potentials. In addition to its conventional antidiabetic activity and emerging antitumor applications, the anti-inflammatory action, weight losing and neuroprotection efficacy of MET has also been extensively investigated for treatment of various diseases (Wang et al. 2017). With the great progression in biomedical nanotechnology, nano-DDS has been widely used for efficient delivery of different types of therapeutic agents for disease treatment. In recent years, a variety of MET-loaded nanocarriers were rationally designed to improve its therapeutic efficacy in anti-inflammatory, weight losing and neuroprotection. In this section, we will briefly discuss the recent trends in nano-DDS of MET for other therapeutic aims apart from its antidiabetic and antitumor activity.

\section{Nano-DDS of MET for anti-inflammatory applications}

Evidences have shown that that MET exerted favorable anti-inflammatory effects by the activation of AMP protein kinase (AMPK) and inhibition of NF-KB (Pereira et al. 2018). Formulating MET into suitable nanocarriers could significantly improve its therapeutic efficacy (Pereira et al. 2018). For instance, Pereira et al. prepared a MET-loaded nano-DDS by encapsulating it in poly(D,L-lactide-co-glycolide) (PLGA) NPs for treatment of periodontal disease 
in diabetic rat model (Pereira et al. 2018). Oral administration of MET-loaded PLGA NPS improved therapeutic efficiency by reducing inflammation and bone loss in periodontitis in diabetic rats (Pereira et al. 2018). Additionally, rational design of guided bone regeneration membrane is of crucial importance for maintaining the enough space for bone regeneration (Zhu et al. 2020). MET was found to have the capacity for facilitate osteogenic differentiation of bone mesenchymal stem cells (Zhu et al. 2020). In order to further improve the osteoinductive ability of MET, a MET-encapsulated polycaprolactone/chitosan nanofibrous membrane was prepared by using electrospinning technique and glutaraldehyde crosslinking method (Zhu et al. 2020). MET released from the nanofibrous membrane showed a sustained release behavior during 23 days (Zhu et al. 2020). More importantly, the prepared MET-loaded membrane exhibited advantages in cell adhesion, proliferation, and osteogenic differentiation of bone mesenchymal stem cells, resulting in good osteoinductive ability in bone regeneration (Zhu et al. 2020).

\section{Nano-DDS of MET for fat controlling and weight reducing}

Moreover, the weight reducing function of MET via modulation of lipid metabolism has also attracted more and more attention due to growing serious obesity problems (Abdellatif and Tawfeek 2016). It's widely accepted that the intraabdominal visceral and subcutaneous fat were highly related to obesity (Abdellatif and Tawfeek 2016). To address this problem, Abdellatif et al. prepared a MET-loaded carbopol gel using carbopol 934, in order to achieve the local delivery of the drug to the abdomen area for lowering the intra-abdominal visceral fat (Abdellatif and Tawfeek 2016). Compared with MET tablets and control (placebo) groups, topical delivery of MET-loaded carbopol gel demonstrated distinct advantages in decreasing the diameter of the abdomen (Abdellatif and Tawfeek 2016). In addition, nonalcoholic fatty liver disease (NAFLD) also represents a main cause for liver diseases (Zai et al. 2019). Interleukin-22 (IL22) has been utilized to treat NAFLD, but its therapeutic efficacy is greatly impeded by its narrow therapeutic window (Zai et al. 2019). To improve the therapeutic efficiency of IL-22, Zai et al. synthesized a polymeric derivative of MET by conjugating it on chitosan, and the polymeric derivative of MET could be utilized as a versatile carrier material for efficient gene delivery (Zai et al. 2019). More importantly, the polymeric derivative of MET inherited the intrinsic therapeutic efficacy from MET against NAFLD (Zai et al. 2019). An IL-22 gene-encapsulated nanocomplex was prepared by mixing the polymeric derivative of MET, penetratin and DSPE-PEG2000 based on the electrostatic interactions (Zai et al. 2019). After intravenous administration, the nanocomplex showed advantages in cellular uptake, endosomal escape, biocompatibility as well as liver accumulation, resulting in satisfactory therapeutic efficacy in the treatment of NAFLD (Zai et al. 2019).

\section{Nano-DDS of MET for neuroprotection}

Additionally, MET has also been found to possess the therapeutic activity in the treatment of Parkinson's disease (Sardoiwala et al. 2020). It has been found that Parkinson's disease was highly correlated with the agglomeration of $\alpha$-synuclein $(\alpha-S y n)$, oxidative stress, mitochondrial dysfunction and deficiency of dopaminergic neurons (Sardoiwala et al. 2020). The neuroprotective effect of MET is contributed to its anti-inflammatory action, induction of autophagy and reduction of phosphorylated proteins involved agglomeration of $\alpha$-synuclein (Sardoiwala et al. 2020). Recently, Sardoiwala et al. prepared a MET-loaded polydopamine nano-DDS for Parkinson's disease therapy by virtue of neuroprotection efficacy of MET (Sardoiwala et al. 2020). The neuroprotective efficiency of MET-loaded polydopamine nano-DDS was found to be related to downregulation of $\alpha$-Syn, reduction in oxidative stress, prevention of apoptosis and anti-inflammatory effects (Sardoiwala et al. 2020). Therefore, MET-loaded polydopamine nano-DDS showed great potential in the prevention and treatment of Parkinson's disease via the neuroprotective activity, which provides a promising nanotherapeutic approach for possible clinical applications in the future.

\section{Conclusion}

MET, a conventional drug, has been found to possess multiple biological activities instead of being limited to antidiabetic applications. With a cationic biguanide main structure, the transmembrane transport of MET is highly dependent on the OCTs, which would not only greatly limit oral absorption efficiency but also restrict its therapeutic efficacy against other diseases. Formulating MET into nano-DDS could significantly change its physicochemical properties and in vivo delivery characteristics. Recently, great progression has been made in nano-DDS of MET, and a variety of nanocarriers have been constructed to improve the delivery efficacy of MET. The present review outlines the recent developments in advanced nano-DDS of MET, including nano-DDS of MET for diabetes therapy, nano-DDS of MET for anticancer treatment, and nano-DDS of MET for other therapeutic aims (e.g. anti-inflammatory, losing fat and neuroprotection).

Despite the great progression in nano-DDS of MET, several important issues should be fully taken into consideration for the success clinical translation of these emerging nano-formulations. First, formulating MET into nanocarriers will inevitably raise the manufacturing cost and increase the 
economic burdens of patients, especially for those diabetic patients on long-term medication. Second, several METbased carrier materials were designed for efficient drug delivery or combination treatments, their biocompatibility and long-term toxicity should be further investigated. Then, the nanostructure of some nanocarriers is relatively complicated, which might make it difficult to scale-up preparation. Finally, the species gap between human beings and animal models should also be a main factor affecting the clinical translation of nano-DDS of MET. Therefore, there's still a long way to truly put the conventional drug in new applications.

Acknowledgements This work was financially supported by the National Science and Technology Major Projects for Major New Drugs Innovation and Development (No. 2017ZX09101-001-005), the National Natural Science Foundation of China (No. 81703451) and the Science and Technology Plan Project of Shenyang (No. 18-400-4-08).

\section{Compliance with ethical standards}

Conflict of interest The authors declare that they have no conflict of interest.

Research involving human and animal rights This article does not contain any studies with human and animal subjects performed by any of the authors.

Open Access This article is licensed under a Creative Commons Attribution 4.0 International License, which permits use, sharing, adaptation, distribution and reproduction in any medium or format, as long as you give appropriate credit to the original author(s) and the source, provide a link to the Creative Commons licence, and indicate if changes were made. The images or other third party material in this article are included in the article's Creative Commons licence, unless indicated otherwise in a credit line to the material. If material is not included in the article's Creative Commons licence and your intended use is not permitted by statutory regulation or exceeds the permitted use, you will need to obtain permission directly from the copyright holder. To view a copy of this licence, visit http://creativecommons.org/licenses/by/4.0/.

\section{References}

Abdellatif A, Tawfeek HM (2016) Metformin loaded carbopol gel for lowering the intra-abdominal visceral fat. J Bioequiv Availab $8: 149-152$

Aldea M, Florian IS, Potara M, Soritau O, Nagy-Simon T, Kacso G (2018) Metformin delivery using chitosan-capped gold nanoparticles in glioblastoma cell lines. Roman Neurosurg 32(2):230-239

Arafa K, Shamma RN, El-Gazayerly ON, El-Sherbiny IM (2018) Facile development, characterization, and optimization of new metformin-loaded nanocarrier system for efficient colon cancer adjunct therapy. Drug Dev Ind Pharm 44(7):1158-1170

Baker NC, Ekins S, Williams AJ, Tropsha A (2018) A bibliometric review of drug repurposing. Drug Discov Today 23:661-672

Banala VT, Sharma S, Barnwal P, Urandur S, Shukla RP, Ahmad N, Mittapelly N, Pandey G, Dwivedi M, Kalleti N, Mitra K, Rath SK, Trivedi R, Mishra PR (2018) Synchronized ratiometric codelivery of metformin and topotecan through engineered nanocarrier facilitates in vivo synergistic precision levels at tumor site. Adv Healthc Mater 7(19): 1800300

Bhujbal S, Dash AK (2018) Metformin-loaded hyaluronic acid nanostructure for oral delivery. AAPS PharmSciTech 19(6):2543-2553

Chastain CA, Klopfenstein N, Serezani CH, Aronoff DM (2019) A clinical review of diabetic foot infections. Clin Podiatr Med Surg 36:381-395

Chaudhury A, Duvoor C, Reddy Dendi VS, Kraleti S, Chada A, Ravilla R, Marco A, Shekhawat NS, Montales MT, Kuriakose K, Sasapu A, Beebe A, Patil N, Musham CK, Lohani GP, Mirza W (2017) Clinical review of antidiabetic drugs: implications for type 2 diabetes mellitus management. Front Endocrinol 8:6

Chen Q, Liu G, Liu S, Su H, Wang Y, Li J, Luo C (2018) Remodeling the tumor microenvironment with emerging nanotherapeutics. Trends Pharmacol Sci 39:59-74

Cui Y, Shan W, Zhou R, Liu M, Wu L, Guo Q, Zheng Y, Wu J, Huang Y (2018) The combination of endolysosomal escape and basolateral stimulation to overcome the difficulties of "easy uptake hard transcytosis" of ligand-modified nanoparticles in oral drug delivery. Nanoscale 10(3):1494-1507

Farajzadeh R, Pilehvar-Soltanahmadi Y, Dadashpour M, Javidfar S, Lotfi-Attari J, Sadeghzadeh H, Shafiei-Irannejad V, Zarghami N (2018) Nano-encapsulated metformin-curcumin in PLGA/PEG inhibits synergistically growth and hTERT gene expression in human breast cancer cells. Artif Cell Nanomed B 46(5):917-925

Gao C, Sun X, Lu L, Liu F, Yuan J (2019) Prevalence of gestational diabetes mellitus in mainland China: a systematic review and meta-analysis. J Diabetes Investig 10:154-162

Javidfar S, Pilehvar-Soltanahmadi Y, Farajzadeh R, Lotfi-Attari J, Shafiei-Irannejad V, Hashemi M, Zarghami N (2018) The inhibitory effects of nano-encapsulated metformin on growth and hTERT expression in breast cancer cells. J Drug Deliv Sci Technol 43:19-26

Jiang T, Chen L, Huang Y, Wang J, Xu M, Zhou S, Gu X, Chen Y, Liang K, Pei Y, Song Q, Liu S, Ma F, Lu H, Gao X, Chen J (2019) Metformin and docosahexaenoic acid hybrid micelles for premetastatic niche modulation and tumor metastasis suppression. Nano Lett 19(6):3548-3562

Jose P, Sundar K, Anjali CH, Ravindran A (2015) Metformin-loaded BSA nanoparticles in cancer therapy: a new perspective for an old antidiabetic drug. Cell Biochem Biophys 71(2):627-636

Kumar CS, Raja MD, Sundar DS, Antoniraj MG, Ruckmani K (2015) Hyaluronic acid co-functionalized gold nanoparticle complex for the targeted delivery of metformin in the treatment of liver cancer (HepG2 cells). Carbohydr Polym 128:63-74

Kumar S, Bhanjana G, Verma RK, Dhingra D, Dilbaghi N, Kim KH (2017) Metformin-loaded alginate nanoparticles as an effective antidiabetic agent for controlled drug release. J Pharm Pharmacol 69(2):143-150

Lee JY, Shin DH, Kim JS (2020) Anticancer effect of metformin in herceptin-conjugated liposome for breast cancer. Pharmaceutics 12(1):11

Li Y, Luo J, Lin MT, Zhi P, Guo WW, Han M, You J, Gao JQ (2019) Co-delivery of metformin enhances the antimultidrug resistant tumor effect of doxorubicin by improving hypoxic tumor microenvironment. Mol Pharmaceut 16(7):2966-2979

Liang X, Giacomini KM (2017) Transporters involved in metformin pharmacokinetics and treatment response. J Pharm Sci 106:2245-2250

Luo C, Wang Y, Chen Q, Han X, Liu X, Sun J, He Z (2012) Advances of paclitaxel formulations based on nanosystem delivery technology. Mini-Rev Med Chem 12(5):434-444

Luo C, Sun J, Du Y, He Z (2014a) Emerging integrated nanohybrid drug delivery systems to facilitate the intravenous-to-oral switch in cancer chemotherapy. J Control Release 176:94-103 
Luo C, Sun J, Sun B, He Z (2014b) Prodrug-based nanoparticulate drug delivery strategies for cancer therapy. Trends Pharmacol Sci 35:556-566

Luo C, Miao L, Zhao Y, Musetti S, Wang Y, Shi K, Huang L (2016a) A novel cationic lipid with intrinsic antitumor activity to facilitate gene therapy of TRAIL DNA. Biomaterials 102:239-248

Luo C, Sun J, Liu D, Sun B, Miao L, Musetti S, Li J, Han X, Du Y, Li L, Huang L, He Z (2016b) Self-assembled redox dual-responsive prodrug-nanosystem formed by single thioether-bridged paclitaxel-fatty acid conjugate for cancer chemotherapy. Nano Lett 16:5401-5408

Luo C, Sun B, Wang C, Zhang X, Chen Y, Chen Q, Yu H, Zhao H, Sun M, Li Z, Zhang H, Kan Q, Wang Y, He Z, Sun J (2019) Selffacilitated ROS-responsive nanoassembly of heterotypic dimer for synergistic chemo-photodynamic therapy. J Control Release 302:79-89

Magdy M, Almahallawi A, Nassar N, Shouman S (2017) Pluronic based cubosomes enhance metformin cytotoxicity in colon cancer cell lines. Clin Ther 39(8):e27

Mirazi N, Shoaei J, Khazaei A, Hosseini A (2015) A comparative study on effect of metformin and metformin-conjugated nanotubes on blood glucose homeostasis in diabetic rats. Eur J Drug Metab Pharmacokinet 40(3):343-348

Mokhtare B, Cetin M, Ozakar RS, Bayrakceken H (2017) In vitro and in vivo evaluation of alginate and alginatechitosan beads containing metformin hydrochloride. Trop J Pharm Res 16(2):287-296

Morales DR, Morris AD (2015) Metformin in cancer treatment and prevention. Annu Rev Med 66:17-29

Nurani M, Akbari V, Taheri A (2017) Preparation and characterization of metformin surface modified cellulose nanofiber gel and evaluation of its anti-metastatic potentials. Carbohydr Polym $165: 322-333$

Osama H, Sayed OM, Hussein RRS, Abdelrahim M, Elberry A (2019) Design, optimization, characterization, and in vivo evaluation of sterosomes as a carrier of metformin for treatment of lung cancer. J Liposome Res. https://doi.org/10.1080/08982104.2019.1610434

Pakkir Maideen NM, Jumale A, Balasubramaniam R (2017) Drug interactions of metformin involving drug transporter proteins. Adv Pharm Bull 7:501-505

Patiño-Herrera R, Louvier-Hernández JF, Escamilla-Silva EM, Chaumel J, Escobedo AGP, Pérez E (2019) Prolonged release of metformin by $\mathrm{SiO}_{2}$ nanoparticles pellets for type II diabetes control. Eur J Pharm Sci 131:1-8

Peng M, Darko KO, Tao T, Huang Y, Su Q, He C, Yin T, Liu Z, Yang $X$ (2017) Combination of metformin with chemotherapeutic drugs via different molecular mechanisms. Cancer Treat Rev 54:24-33

Pereira ADSBF, Brito GADC, Lima MLDS et al (2018) Metformin hydrochloride-loaded PLGA nanoparticle in periodontal disease experimental model using diabetic rats. Int J Mol Sci 19(11):3488

Qian RC, Lv J, Li HW, Long YT (2017) Sugar-coated nanobullet: growth inhibition of cancer cells induced by metformin-loaded glyconanoparticles. ChemMedChem 12(22):1823-1827

Ramasamy T, Ruttala HB, Kaliraj K, Poudel K, Giu S, Jin Choi HG, Ku SK, Yong CS, Kim JO (2019) Polypeptide derivative of metformin with the combined advantage of a gene carrier and anticancer activity. Acs Biomater Sci Eng 5(10):5159-5168

Sahu AK, Verma A (2016) Development and statistical optimization of chitosan and eudragit based gastroretentive controlled release multiparticulate system for bioavailability enhancement of metformin $\mathrm{HCl}$. J Pharm Investig 46(3):239-252

Samuel VP, Dahiya R, Singh Y, Gupta G, Sah SK, Gubbiyappa SK, Chellappan DK, Dua K (2019) Metformin: a calutary candidate for colorectal cancer treatment in patients with diabetes. J Environ Pathol Toxicol Oncol 38:133-141

Sardoiwala MN, Srivastava AK, Kaundal B, Karmakar S, Choudhury SR (2020) Recuperative effect of metformin loaded polydopamine nanoformulation promoting EZH2 mediated proteasomal degradation of phospho- $\alpha$-synuclein in Parkinson's disease model. Nanomed-Nanotechnol 24:102088

Sarwar MS, Ghaffar A, Islam A, Yasmin F, Oluz Z, Tuncel E, Duran H, Qaiser AA (2020) Controlled drug release behavior of metformin hydrogen chloride from biodegradable films based on chitosan/poly (ethylene glycol) methyl ether blend. Arab J Chem 13(1):393-403

Shafiei-Irannejad V, Samadi N, Salehi R, Yousefi B, Rahimi M, Akbarzadeh A, Zarghami N (2018) Reversion of multidrug resistance by co-encapsulation of doxorubicin and metformin in poly (lactideco-glycolide)-d- $\alpha$-tocopheryl polyethylene glycol 1000 succinate nanoparticles. Pharm Res 35(6): 119

Shan X, Li S, Sun B, Chen Q, Sun J, He Z, Luo C (2020) Ferroptosisdriven nanotherapeutics for cancer treatment. J Control Release 319:322-332

Shariatinia Z, Zahraee Z (2017) Controlled release of metformin from chitosan-based nanocomposite films containing mesoporous MCM-41 nanoparticles as novel drug delivery systems. J Colloid Interface Sci 501:60-76

Shi K, Zhao Y, Miao L, Satterlee A, Haynes M, Luo C, Musetti S, Huang L (2017) Dual functional lipomet mediates envelopetype nanoparticles to combinational oncogene silencing and tumor growth inhibition. Mol Ther 25(7):1567-1579

Shukla SK, Kulkarni NS, Chan A, Parvathaneni V, Farrales P, Muth A, Gupta V (2019) Metformin-encapsulated liposome delivery system: an effective treatment approach against breast cancer. Pharmaceutics 11(11):559

Shurrab NT, Arafa ESA (2020) Metformin: a review of its therapeutic efficacy and adverse effects. Obes Med 17:100186

Siegel RL (2020) Miller KD and Jemal A (2020) Cancer statistics. CA-Cancer J Clin 70(1):7-30

Song X, Feng L, Liang C, Gao M, Song G, Liu Z (2017) Liposomes co-loaded with metformin and chlorin e6 modulate tumor hypoxia during enhanced photodynamic therapy. Nano Res 10(4):1200-1212

Sun B, Luo C, Yu H, Zhang X, Chen Q, Yang W, Wang M, Kan Q, Zhang H, Wang Y, He Z, Sun J (2018) Disulfide bond-driven oxidation- and reduction-responsive prodrug nanoassemblies for cancer therapy. Nano Lett 18:3643-3650

Sun B, Chen Y, Yu H, Wang C, Zhang X, Zhao H, Chen Q, He Z, Luo C, Sun J (2019a) Photodynamic PEG-coated ROS-sensitive prodrug nanoassemblies for core-shell synergistic chemo-photodynamic therapy. Acta Biomater 92:219-228

Sun B, Luo C, Zhang X, Guo M, Sun M, Yu H, Chen Q, Yang W, Wang M, Zuo S, Chen P, Kan Q, Zhang H, Wang Y, He Z, Sun J (2019b) Probing the impact of sulfur/selenium/carbon linkages on prodrug nanoassemblies for cancer therapy. Nat Commun 10:3211

Sun Y, Zhao D, Wang G, Wang Y, Cao L, Sun J, Jiang Q, He Z (2020) Recent progress of hypoxia-modulated multifunctional nanomedicines to enhance photodynamic therapy: opportunities, challenges, and future development. Acta Pharm Sin B. https://doi.org/10.1016/j.apsb.2020.01.004

Taghizadehghalehjoughi A, Hacimuftuoglu A, Cetin M, Ugur AB, Galateanu B, Mezhuev Y, Okkay U, Taspinar N, Taspinar M, Uyanik A, Gundogdu B, Mohammadzadeh M, Nalci KA, Stivaktakis P, Tsatsakis A, Jung TW, Jeong JH, El-Aty AMA (2018) Effect of metformin/irinotecan-loaded poly-lactic-co-glycolic acid nanoparticles on glioblastoma: in vitro and in vivo studies. Nanomedicine 13(13):1595-1606

van der Meel R, Sulheim E, Shi Y, Kiessling F, Mulder WJM, Lammers T (2019) Smart cancer nanomedicine. Nat Nanotechnol 14:1007-1017 
Vllasaliu D, Thanou M, Stolnik S, Fowler R (2018) Recent advances in oral delivery of biologics: nanomedicine and physical modes of delivery. Expert Opin Drug Deliv 15:759-770

Wang YW, He SJ, Feng X, Cheng J, Luo YT, Tian L, Huang Q (2017) Metformin: a review of its potential indications. Drug Des Dev Ther 11:2421-2429

Xiao Y, Wang S, Zong Q, Yin Z (2018) Co-delivery of metformin and paclitaxel via folate-modified $\mathrm{pH}$-sensitive micelles for enhanced anti-tumor efficacy. AAPS PharmSciTech 19(5):2395-2406

Xiong Y, Zhao Y, Miao L, Lin CM, Huang L (2016) Co-delivery of polymeric metformin and cisplatin by self-assembled coremembrane nanoparticles to treat non-small cell lung cancer. $\mathrm{J}$ Control Release 244:63-73

Yu W, Jiang G, Zhang Y, Liu D, Xu B, Zhou J (2017) Near-infrared light triggered and separable microneedles for transdermal delivery of metformin in diabetic rats. J Mater Chem B 5(48):9507-9513

Yu H, Zhong X, Gao P, Shi J, Wu Z, Guo Z, Wang Z, Song Y (2019) The potential effect of metformin on cancer: an umbrella review. Front Endocrinol 10:617
Zai W, Chen W, Wu Z, Jin X, Fan J, Zhang X, Luan J, Tang S, Mei X, Hao Q, Liu H, Liu H, Ju D (2019) Targeted interleukin-22 gene delivery in the liver by polymetformin and penetratin-based hybrid nanoparticles to treat nonalcoholic fatty liver disease. ACS Appl Mater Interaces 11(5):4842-4857

Zhang M, Chen X, Li C, Shen X (2019) Charge-reversal nanocarriers: an emerging paradigm for smart cancer nanomedicine. J Control Release 319:46-62

Zhao Y, Wang W, Guo S, Wang Y, Miao L, Xiong Y, Huang L (2016) PolyMetformin combines carrier and anticancer activities for in vivo siRNA delivery. Nat Commun 7(1):1-9

Zhu J, Ye H, Deng D, Li J, Wu Y (2020) Electrospun metformin-loaded polycaprolactone/chitosan nanofibrous membranes as promoting guided bone regeneration membranes: Preparation and characterization of fibers, drug release, and osteogenic activity in vitro. J Biomater Appl 3:1282

Publisher's Note Springer Nature remains neutral with regard to jurisdictional claims in published maps and institutional affiliations. 